International Journal of Pure and Applied Mathematics

Volume 110 No. 3 2016, 547-562

ISSN: $1311-8080$ (printed version); ISSN: 1314-3395 (on-line version)

url: http://www.ijpam.eu

doi: 10.12732/ijpam.v110i3.13

ijpam.eu

\title{
OSCILLATORY AND ASYMPTOTIC PROPERTIES OF NONLINEAR FIRST ORDER DIFFERENTIAL EQUATIONS \\ WITH PIECEWISE CONSTANT ARGUMENT OF GENERALIZED TYPE
}

\author{
Todor Kostadinov ${ }^{1}$, Valentina Proycheva ${ }^{2}$ \\ ${ }^{1,2}$ Faculty of Mathematics \\ Technical University of Sofia, Filial Plovdiv \\ 25 Tsanko Dystabanov, 4000, Plovdiv, BULGARIA
}

\begin{abstract}
In the paper, we consider differential equations of neutral type with piecewise constant argument of generalized type, i.e., the argument is a general step function. Sufficient conditions for oscillation of all solutions of this type equations are obtained. The asymptotic behavior of the nonoscillating solutions is studied also. Appropriate examples are given to illustrate the recieved results.
\end{abstract}

Key Words: Neutral type, non oscillatory and oscillatory solution, piecewise constant argument

\section{Introduction}

In his famous survey, A. D. Myshkis [17]among the functional differential equations first give several reasonable occasions to study differential equations with piecewise argument (in short DEPCA). Generally speaking they are equations with hybrid properties, which combine partial the properties of continuous systems, also part of discrete equations properties. Later with the works of J.

Received: $\quad$ April 19, 2016

Revised: October 11, 2016

Published: November 5, 2016

$\S_{\text {Correspondence author }}$ (c) 2016 Academic Publications, Ltd. url: www.acadpubl.eu 
Wiener at all [21], [2], [23] and [24] was started investigation of scalar DEPCA of the type

$$
\frac{d x(t)}{d t}=f(t, x(t), x([t])
$$

where [. ] signifies the greates integer function. This investigation has been extended by many authors: [1], [2], [7], [8], [5], [15], [22] and [6] which study generally the existing of periodic solutions and the oscillatory properties of DEPCA. Some real applications of DEPCA can be found in [4], [8] and [12]. Akhmet in [3] first introduce the function "gama" in his consideration of only delayed situation. This function is widely used in several works of Chiu and Pinto [6], [7] and [8]. In [19] and [20] Pinto has cleared the importance of the advanced and delayed intervals. Some specific results are given in [23], [10] and [7]. Note that to our knowledge almost all investigations are devoted for equations of retarded type. The aim of this work is to extend part of the results investigated by not many autors [15] and [18] for the neutral case.

In the present paper the equation

$$
(y(t)+p y(t+\tau))^{\prime}=q f(y(\gamma(t)))
$$

is investigated, where $p \in R \backslash 0, \tau>0, q<0$ and $\gamma(t)=[t]$ or $\gamma(t)=\left[\frac{t+1}{2}\right]$. The full definition of $\gamma(t)$ will be given below. The alternative case $q>0$ is considered in Oscillatory and Asymptotic properties of Nonlinear First Order Differential Equations with Piecewise Constant Argument of Generalized Type part 2 to be appeared.

\section{Preliminaries}

In the present paper the equation

$$
(y(t)+p y(t+\tau))^{\prime}=q f(y(\gamma(t))
$$

is investigated, where $p \in R \backslash\{0\}$ and $q<0$ (the case $q>0$ is considered in the second part of this paper, to be appeared). Introduce two real sequences $\left\{t_{n}\right\},\left\{\gamma_{n}\right\} n=0,1,2 \ldots$ such that $t_{0}=-\tau, t_{n+1}=t_{n}+1$ and $n<\gamma_{n} \leq t_{n+1}$, $\gamma_{n+1}=\gamma_{n}+1$ where $\gamma(t)$ is a step function given by

$$
\gamma(t)= \begin{cases}{[t],} & t_{n} \leq t<\gamma_{n} \\ 2\left[\frac{t+1}{2}\right], & \gamma_{n} \leq t<t_{n+1}\end{cases}
$$


The function $\gamma(t)$ can be rewrited in the form

$$
\gamma(t)= \begin{cases}0, & t \in\left[-\frac{1}{2}, \frac{1}{2}\right), \\ n-1, & t \in\left[n-\frac{1}{2}, n\right), \\ n, & t \in\left[n, \gamma_{n}\right), \\ n+1, & t \in\left[\gamma_{n}, n+\frac{1}{2}\right),\end{cases}
$$

$n=1,2,3 \ldots$. Note that the equation (1) is of advanced type in $I_{n}^{+}=\left[t_{n}, \gamma_{n}\right)$ and of retarded type in $I_{n}^{-}=\left[\gamma_{n}, t_{n+1}\right)$. This decomposition will be present in all our results.

As usual with $C$ we denote the Banach space of initial functions $C=$ $\varphi:[-\tau, 0] \rightarrow R: \varphi \in C([-\tau, 0], R)$ with norm $\|\varphi\|=\sup t \in[-\tau, 0]|\varphi(t)|$ and introduce the initial condition:

$$
y(t)=\varphi(t), t \in[-\tau, 0], \varphi \in C .
$$

Definition 1. The function $y(t)$ is said to be a solution of initial value problem $(I V P)(1),(2)$ if:

(a) $y(t) \in C([-\tau, \infty), R)$ and satisfy the condition (2) for some $\varphi \in C$.

(b) $z(t)=y(t)+p y(t+\tau)$ has a derivate for each $t \in[-\tau, \infty)$ with an exeption of a points of form $t=\gamma_{n}$, and $t=n n=0,1,2 \ldots$ at which there exist right-side derivates.

(c) $y(t)$ satisfies equation (1) in each interval $[n-\tau, n-\tau+1) n=0,1,2 \ldots$.

It is not difficult to chek that for arbitrary initial function the $I V P(1),(2)$ has a unique solution (see Chapter 3 [16], Chapters 2 and 3 [14]).

Definition 2. The solution of equation (1) is said to oscillate if the set of its zeros is unbounded above, otherwise it is called nonoscillating.

Definition 3. The function $f$ is said to finally enjoy the property $K$ if there exists $t_{0}$ such that for $t \geq t_{0}, f$ enjoys the property $K$.

We shall say that conditions $(A)$ are satisfied if the following conditions hold:

A1. $f \in C(R, R)$;

A2. $u f(u)>0$ for $u \neq 0$;

A3. For any $\delta>0$ there exists $\bar{\delta}>0$ such that if $|u|>\delta$ then $|f(u)|>\bar{\delta}$.

Lemma 1. Let conditions $(A)$ hold and let $y(t)$ be a nonoscillating solution of equation (1). Then the following assertions are valid: 
(a) If there exists the finite limit $\lim _{t \rightarrow \infty} z(t)$, then $\liminf _{t \rightarrow \infty}|y(t)|=0$.

(b) If $p<0$, then $\liminf _{t \rightarrow \infty} y(t)=0$ or $\lim _{t \rightarrow \infty}|y(t)|=\infty$.

Proof. a1) Let $t \in[n-\tau, n-\tau+1) n=1,2,3 \ldots$. Then equation (1) can be written in the form

$$
z^{\prime}(t)=q f(y(\gamma(t))) .
$$

Integrate the above equation from $n-\tau$ to $t$ and obtain

$$
z(t)-z(n-\tau)=q f(y(n-1))(t-n+\tau) .
$$

Since $y(t) \in C([-\tau, \infty), R)$ then $z(t)$ is a continious function. By passing to the limit as $t \rightarrow n$ we obtain

$$
z(n)-z(n-\tau)=\frac{q}{2} \tau f(y(n-1)) .
$$

Since there exists $\lim _{t \rightarrow \infty} z(t)=c$ when $n \rightarrow \infty$ then $\lim _{n \rightarrow \infty} f(y(n-1))=0$ and from A3 it follows $\lim _{n \rightarrow \infty} y(n)=0$ and $\liminf _{t \rightarrow \infty}|y(t)|=0$.

b) Let $y(t)>0$. As $q<0$ then $z(t)$ is a decreasing function. Suppose that $\lim _{t \rightarrow \infty} z(t)=c(c>-\infty)$. Then from (a) it follows that $\liminf _{t \rightarrow \infty} y(t)=0$.

Let $\lim _{t \rightarrow \infty} z(t)=-\infty$. Then there exists a number $n_{0}$ such that

$$
z(t) \leq \min _{s \in[t-1, t+1]} z(s)<0,
$$

for $t \in\left[n_{0}, \infty\right)$ and then

$$
y(t) \geq \frac{\min _{s \in[t-1, t+1]}}{p}>0 .
$$

Therefore we have that $\lim _{t \rightarrow \infty} y(t)=\infty$.

In the same way assertion (b) is proved in the case when the nonoscillating solution $y(t)$ is negative.

Introduce the notation $y(n)=c_{n}, n=0,1,2, \ldots$.

Let $t \in\left[n-\frac{1}{2}, n+\frac{1}{2}\right) n=1,2,3 \ldots$. Integrate equation (1) from $n$ to $t$ for $\tau=\frac{1}{2}$ and obtain

$$
y(t)+p y\left(t+\frac{1}{2}\right)=c_{n}+p y\left(n+\frac{1}{2}\right)+q f\left(c_{n}\right) \gamma_{0}+q f\left(c_{n+1}\right)\left(t-\gamma_{n}\right) .
$$


From equation (6) and the continuity of $y(t)$, passing to the limit as $t \rightarrow$ $n+\frac{1}{2}$ the equality

$$
(1-p) y\left(n+\frac{1}{2}\right)=c_{n}-p c_{n+1}+q f\left(c_{n}\right) \gamma_{0}+q\left(\frac{1}{2}-\gamma_{0}\right) f\left(c_{n+1}\right)
$$

follows.

We shall consider separatelly the cases $p=1$ and $p \neq 1$. Let $p=1$. Then from equation (7) it follows that if $y(t)$ is solution of equation (1) the sequence $\left\{c_{n}\right\}_{n=1}^{\infty}$ satisfies the difference equations:

$$
c_{n+1}-c_{n}=q \gamma_{0} f\left(c_{n}\right)+q\left(\frac{1}{2}-\gamma_{0}\right) f\left(c_{n+1}\right)
$$

when $t \in\left[n-\frac{1}{2}, n+\frac{1}{2}\right), n=1,2,3 \ldots$

Let $p \neq 1$. Let $t \in\left[n+\frac{1}{2}, n+\frac{3}{2}\right)$. As in the above, we obtain the equality

$$
y\left(n+\frac{3}{2}\right)=\frac{1}{1-p}\left(c_{n+1}-p c_{n+2}+q \gamma_{0} f\left(c_{n+1}\right)+q\left(\frac{1}{2}-\gamma_{0}\right) f\left(c_{n+2}\right)\right) .
$$

On the other hand integrating from $n+1$ to $t$ we obtain

$$
y(t)+p y\left(t+\frac{1}{2}\right)=c_{n+1}+p y\left(n+\frac{3}{2}\right)+q \gamma_{0} f\left(c_{n+1}\right)+q f\left(c_{n+2}\right)\left(t-\gamma_{n+1}\right) .
$$

For $t \rightarrow n+\frac{1}{2}$, we obtain

$$
y\left(n+\frac{1}{2}\right)+p c_{n+1}=c_{n+1}+p y\left(n+\frac{3}{2}\right)+q \gamma_{0} f\left(c_{n+1}\right)-q\left(\frac{1}{2}+\gamma_{0}\right) f\left(c_{n+2}\right) \text {. }
$$

Substituting $y\left(n+\frac{1}{2}\right)$ and $y\left(n+\frac{3}{2}\right)$ from equation (7) and equation (9) it follows

$$
\begin{aligned}
& c_{n+2}-c_{n+1}+\frac{1}{p^{2}} c_{n}-\frac{1}{p^{2}} c_{n+1}+\frac{q \gamma_{0}}{p^{2}} f(\left.c_{n}\right)+\frac{1}{p^{2}}\left(\frac{q}{2}-2 q \gamma_{0}\right) f\left(c_{n+1}\right) \\
&+\frac{1}{p^{2}}\left(\frac{q}{2}+q \gamma_{0}-p q\right) f\left(c_{n+2}\right)=0 .
\end{aligned}
$$

Hence for $p \neq 1$ if equation (1) has a solution $y(t)$, then the sequence $\left\{c_{n}\right\}_{n=1}^{\infty}$ satisfies the difference equation equation (11). 


\section{Main Results}

We shall consider the asympthotic behavior of the nonoscillating solution of equation (1). We shall first consider the case when $q<0$. The case $q>0$ is considered in the second part of this paper which will be published later.

Theorem 1. Let conditions $(A)$ hold, $q<0$ and $p<-1$. Then, if $y(t)$ is a nonoscillating solution of equation (1), $\liminf _{t \rightarrow \infty} y(t)=0$ or $\lim _{t \rightarrow \infty}|y(t)|=\infty$. Moreover when $\lim _{t \rightarrow \infty} z(t) \geq 0$ then $\lim t \rightarrow \infty y(t)=0$.

Proof. Let $y(t)>0$ finally. Then $z(t)$ is a finally decreasing function and by Lemma $1, \liminf _{t \rightarrow \infty} y(t)=0$ or $\lim _{t \rightarrow \infty} y(t)=\infty$

Let $\lim _{t \rightarrow \infty} z(t) \geq 0$. Since $z(t)$ is a finally decreasing function, then $z(t)>$ Ofinally, i.e. $y(t)+p y(t+\tau)>0$. Hence $y(t)>-p y(t+\tau)>y(t+\tau)$. From it immediatelly follows that $y(t)$ is a bounded function. Let $\lim \sup y(t)=d$. Suppose that $d>0$. Choose the sequance $\left\{t_{n}\right\}_{n=1}^{\infty}$ so that $\lim _{n \rightarrow \infty}^{t \rightarrow \infty} t_{n}=\infty$ and $\lim _{n \rightarrow \infty} y(n+\tau)=d$. Consider the sequance $\left\{y\left(t_{n}\right)\right\}_{n=1}^{\infty}$. Let $\limsup _{n \rightarrow \infty} y\left(t_{n}\right)=$ $c$ (obviously $c \leq d$ ). Choose a subsequance $\left\{n_{k}\right\}_{k=1}^{\infty}$ such that $n_{k} \geq k$ and $\lim _{k \rightarrow \infty} y\left(t_{n_{k}}\right)=c$. Then

$$
\lim _{k \rightarrow \infty} z\left(t_{n_{k}}\right)=c+p d \leq(1+p) d<0 .
$$

The contradiction obtained shows that $\limsup _{t \rightarrow \infty} y(t)=0$ and $\lim _{t \rightarrow \infty} y(t)=0$. The case $y(t)<0$ is considered analogously.

Theorem 2. Let conditions $(A)$ hold, $q<0$ and $p \in(-1,0)$. Then for each bounded nonoscillating solution of equation (1) $\lim _{t \rightarrow \infty} z(t)=c(c \geq 0)$ if $y(t)>0$ finally or $\lim _{t \rightarrow \infty} z(t)=c(c \leq 0)$ if $y(t)<0$.

Proof. Let $y(t)$ be a finally positive solution of equation (1)and $\lim _{t \rightarrow \infty} z(t) \leq$ 0 . By Lemma 1 since $y(t)$ is a bounded function, then $\liminf _{t \rightarrow \infty} y(t)=0$. Let $\limsup y(t)=d$. Suppose $d>0$. Choose a sequance $\left\{t_{n}\right\}_{n=1}^{\infty}$, such that $\lim _{n \rightarrow \infty}^{t \rightarrow \infty} t_{n}=\infty$ and $\lim _{n \rightarrow \infty} y\left(t_{n}\right)=d$. Let $\limsup _{n \rightarrow \infty} y\left(t_{n}+\tau\right)=c$ (obviously $\left.c \leq d\right)$. Choose a sequance $\left\{n_{k}\right\}_{k=1}^{\infty}\left(n_{k} \geq k\right)$ such that $\lim _{k \rightarrow \infty} y\left(t_{n_{k}}+\tau\right)=c$. Then

$$
\lim _{k \rightarrow \infty} z\left(t_{n_{k}}\right)=\lim _{k \rightarrow \infty}\left(y\left(t_{n_{k}}\right)+p y\left(t_{n_{k}}+\tau\right)\right)=d+p c \geq d(1+p)>0 .
$$


The contradiction obtained shows that $\limsup _{t \rightarrow \infty} y(t)=0$ and $\lim _{t \rightarrow \infty} y(t)=0$. This fact implies imediately that $\lim _{t \rightarrow \infty} z(t)=0$. Analogously it is proved the case when $y(t)<0$ finally.

Remark 1. Theorem 2 naturally sets the question:What can we claim about the unbounded solutions of equation (1) when $p \in(-1,0)$ and whether it is true that, for each unbounded solution $y(t)$ of equation $(1), \lim _{t \rightarrow \infty}|y(t)|=\infty$ ? The answer to this question is negative. We shall show this by means of the following example:

Example 1. Consider the equation

$$
\left(y(t)-\frac{1}{2} y\left(t+\frac{1}{2}\right)\right)^{\prime}=-\frac{1}{4} y(\gamma(t))
$$

with $\gamma_{0}=\frac{1}{2}$ and with initial function

$$
\varphi(t)= \begin{cases}\frac{13}{2}+\frac{1}{2 \lambda(1-\lambda)}-\frac{1}{4(1-\lambda)}+\left(12-\frac{1}{1-\lambda}\right) t, & t \in\left[-\frac{1}{2},-\frac{1}{4}\right) \\ 1-\left(15+\frac{1}{4 \lambda(1-\lambda)}\right) t, & t \in\left[-\frac{1}{4}, 0\right]\end{cases}
$$

where $\lambda$ is the root of the equation $F(\lambda)=4 \lambda^{3}-22 \lambda^{2}+\frac{31}{2} \lambda+1=0$ which is in the interval $(0,1)$. There exists such a root because $F(0)=\frac{1}{2}>0$ and $F(1)=-\frac{3}{2}<0$.

Define the function $y(t)$ in $\left[-\frac{1}{2}, \infty\right)$ as

$$
y\left(t+\frac{1}{2}\right)=2(y(t)-z(t)), \quad t \in\left[-\frac{1}{2}+\frac{k}{2}, \frac{k}{2}\right], \quad k=0,1,2 \ldots,
$$

where

$$
\begin{gathered}
y(t)=\varphi(t), \quad t \in\left[-\frac{1}{2}, 0\right], \\
z(t)= \begin{cases}\frac{1}{4} \lambda^{n-1}\left[n+\frac{\lambda-\frac{1}{2}}{2(1-\lambda)}\right]-\frac{1}{4} \lambda^{n-1} t, & t \in\left[n-\frac{1}{2}, n\right), \\
\frac{1}{4} \lambda^{n}\left[n+\frac{\lambda-\frac{1}{2}}{2 \lambda(1-\lambda)}\right]-\frac{1}{4} \lambda^{n} t, & t \in\left[n, n+\frac{1}{2}\right),\end{cases}
\end{gathered}
$$

$n=0,1,2, \ldots$

We shall prove that $y(t)$ is a solution of equation (12) with initial function $\varphi(t)$ and that $y(t)>0$ in $\left[-\frac{1}{2}, \infty\right)$. From the definition of the function $y(t)$ it follows that

$$
z(t)=y(t)-\frac{1}{2} y\left(t+\frac{1}{2}\right) .
$$


We shall prove that

$$
z^{\prime}(t)=-\frac{1}{4} y(\gamma(t))
$$

in each interval $\left[n-\frac{1}{2}, n+\frac{1}{2}\right]$. From the definition of $z(t)$ it follows that

$$
\begin{gathered}
z(n)=\frac{1}{4} \frac{\lambda-\frac{1}{2}}{2 \lambda(1-\lambda)} \lambda^{n}, \\
z\left(n+\frac{1}{2}\right)=\frac{1}{4}\left(\frac{\lambda-\frac{1}{2}}{2(1-\lambda)}-\frac{1}{2}\right) \lambda^{n} .
\end{gathered}
$$

We shall prove by induction that $y(n)=\lambda^{n}, n=0,1,2, \ldots$. Since $y(0)=$ $\varphi(0)=1$, the assertion is valid for $n=0$. Let $y(n)=\lambda^{n}$,

$$
\begin{gathered}
z(n)=y(n)-\frac{1}{2} y\left(n+\frac{1}{2}\right), \\
z\left(n+\frac{1}{2}\right)=y\left(n+\frac{1}{2}\right)-\frac{1}{2} y(n+1) .
\end{gathered}
$$

Hence

$$
y(n+1)=4\left(y(n)-z(n)-\frac{1}{2} z\left(n+\frac{1}{2}\right)\right)
$$

or

$$
\begin{aligned}
y(n+1) & =4\left(\lambda^{n}-\frac{1}{4} \frac{\lambda-\frac{1}{2}}{2 \lambda(1-\lambda)} \lambda^{n}-\frac{1}{8}\left(\frac{\lambda-\frac{1}{2}}{2(1-\lambda)}-\frac{1}{2}\right) \lambda^{n}\right) \\
& =4 \lambda^{n}\left(1-\frac{\left(\lambda-\frac{1}{2}\right)}{8 \lambda(1-\lambda)}-\frac{2 \lambda-\frac{3}{2}}{16(1-\lambda)}\right) .
\end{aligned}
$$

Taking into account $\lambda$, we immediatelly verify that

$$
1-\frac{\lambda-\frac{1}{2}}{16 \lambda(1-\lambda)}+\frac{2 \lambda-\frac{3}{2}}{16(1-\lambda)}=\frac{\lambda}{4} .
$$

Then $y(n+1)=\lambda^{n+1}$ and the inductive step is complete. From the equality proved, $y(n)=\lambda^{n}$, and from definition of $z(t)$ it immediately follows that $y(t)$ satisfies equation (12) in each interval $\left[n-\frac{1}{2}, n+\frac{1}{2}\right)$. Since $y(t)$ is a linear function in each interval $\left[-\frac{1}{2}+\frac{k}{4},-\frac{1}{4}+\frac{k}{4}\right], k=0,1,2, \ldots$, then in order to prove that $y(t)>0$ it suffices to prove that $y\left(-\frac{1}{2}+\frac{k}{4}\right)>0$ for $k=0,1,2, \ldots$

$$
y\left(-\frac{1}{2}\right)=\frac{1}{2}+\frac{1}{4(1-\lambda)}>0, \quad y\left(-\frac{1}{2}+\frac{k}{4}\right),
$$


depends on $k$ and is in one of the forms: $y(n), y\left(n+\frac{1}{2}\right)$ and $y\left(-\frac{1}{4}+\frac{n}{2}\right)$.

Obviously, $y(n)>0, n=0,1,2, \ldots$

From equation $(7)$ and (for $f(t)=t$ ) it follows that $y\left(n+\frac{1}{2}\right)=\frac{7}{12} y(n)+$ $\frac{1}{3} y(n+1)>0, n=0,1,2, \ldots$.

It remains to prove that $y\left(-\frac{1}{4}+\frac{n}{2}\right)>0$. The function $z(t)$ is strictly decreasing in $\left[-\frac{1}{2}, \infty\right)$ and $z\left(-\frac{1}{2}\right)=\frac{1}{4(1-\lambda)}<1$. Hence $z(t)<1$ in $\left[-\frac{1}{2}, \infty\right)$. We shall show by induction that $y\left(-\frac{1}{4}+\frac{n}{2}\right)>4$. Since $y\left(-\frac{1}{4}\right)=1+\frac{15}{4}+\frac{1}{16 \lambda(1-\lambda)}>4$, then for $n=0$ the assertion is valid. Let $y\left(-\frac{1}{4}+\frac{n}{2}\right)>4$. The point $-\frac{1}{4}+\frac{n}{2}$ is either in the form $k+\frac{1}{4}$ or of the form $k+\frac{3}{4}$. Suppose that $-\frac{1}{4}+\frac{n}{2}=k+\frac{1}{4}$. Then $y\left(k+\frac{3}{4}\right)=2\left[y\left(k+\frac{1}{4}\right)-z\left(k+\frac{1}{4}\right)\right]>6$. Analogously the case $-\frac{1}{4}+\frac{n}{2}=k+\frac{3}{4}$ is considered. Thus we have established that the function $y(t)$ defined above is a strictly positive(i.e., nonoscillating)solution of equation (12). Since $y(n)=\lambda^{n}$ and $\lambda<1$, then $\liminf _{t \rightarrow \infty} y(t)=0$. We shall prove that $\limsup _{t \rightarrow \infty} y(t)=\infty$.

Consider the sequances $b_{n}=y\left(-\frac{1}{4}+\frac{n}{2}\right)$ and $d_{n}=z\left(-\frac{1}{4}+\frac{n}{2}\right)$. The equality

$$
b_{n+1}=2\left(b_{n}-d_{n}\right)
$$

is valid. Moreover, by virtue of what was proved above $\lim _{n \rightarrow \infty} d_{n}=0$ and $b_{n}>4$. From the estimate $b_{n+1}-b_{n}=b_{n}-2 d_{n}>4-2=2$.

It follows that $\left\{b_{n}\right\}$ is a strictly increasing sequance. Suppose that $\lim _{n \rightarrow \infty} b_{n}=$ $b<\infty$. Passing to the limit in equation (13), we obtain the equality $b=2 b$. But $b_{n}>4$, i.e. $b>4 \neq 0$. The contradiction obtained shows that

$$
\lim _{n \rightarrow \infty} b_{n}=\lim _{n \rightarrow \infty} y\left(-\frac{1}{4}+\frac{n}{2}\right)=\infty
$$

and

$$
\limsup _{t \rightarrow \infty} y(t)=\infty .
$$

Finaly, the solution $y(t)$ of equation (12) constructed above is nonoscillating,

$$
\liminf _{t \rightarrow \infty} y(t)=0 \text { and } \limsup _{t \rightarrow \infty} y(t)=\infty .
$$

Example 1 shows that the behavior of the solutions of equation (1) may be considerably more complex than that of the equations without a piecewise constant argument. For instance, for the neutral equation analogous to equation $(12)$,

$$
\left(y(t)-\frac{1}{2} y\left(t+\frac{1}{2}\right)\right)^{\prime}=-\frac{1}{4} y\left(t+\frac{1}{2}\right) .
$$

It is known that its nonoscillating solutions either tend to 0 or diverge to $\pm \infty$. 
Theorem 3. Let conditions $(A)$ hold, $q<0$ and $p=-1$. Then, if $y(t)$ is an unbounded nonoscillating solution of equation (1), $\lim _{t \rightarrow \infty}|y(t)|=\infty$.

Proof. Let $y(t)$ be an unbounded finally positive solution of equation (1). Suppose that $\lim _{t \rightarrow \infty} z(t) \geq 0$. Since $z(t)$ is a finally strictly decreasing function, then $z(t)>0$ finally. Hence $y(t)-y(t+\tau)>0$ and $y(t)>y(t+\tau)$. The last inequality obviously contradicts the fact that $y(t)$ is an unbounded function. Let $\lim _{t \rightarrow \infty} z(t)=c(c<0)$. It means that $z(t)$ is finally negative and $y(t)<y(t+\tau)$ for enough large $t$. So $y(t)$ is increasing unbounded function hence $\lim _{t \rightarrow \infty} y(t)=\infty$. And in the end if $\lim _{t \rightarrow \infty} z(t)=-\infty$ from the inequality $-y(t+\tau)<z(t)$ we obtain $\lim _{t \rightarrow \infty} y(t)=\infty$. The case when $y(t)$ is an unbounded negative solution of equation (1) is analogous.

Remark 2. In Theorem 3 there is no claim concerning the bounded solutions of equation (1). The reason is the following:From Lemma 1 it follows that if $y(t)$ is a bounded, nonoscillating solution of equation (1), then $\liminf _{t \rightarrow \infty} y(t)=0$. It turns out, however, that equation (1) has solutions $y(t)$ for which $\limsup _{t \rightarrow \infty} y(t)>0$, i.e., for which the limit $\lim _{t \rightarrow \infty} y(t)$ does not exist. In this case (as in Example 1 the behaviour of the nonoscillating solutions of equation (1) is more complex than that of the corresponding equations without a piecewise constant argument. We shall illustrate this fact by the following example.

Example 2. Consider the equation

$$
\left(y(t)-y\left(t+\frac{1}{2}\right)\right)^{\prime}=-y(\gamma(t))
$$

with initial function $\varphi(t)$,

$$
\varphi(t)= \begin{cases}\frac{1-3 \lambda}{2 \lambda^{2}(1-\lambda)}-\frac{1-4 \lambda+\lambda^{2}}{\lambda(1-\lambda)}(4 t+2), & t \in\left[-\frac{1}{2},-\frac{1}{4}\right], \\ \frac{-2 \lambda^{3}+8 \lambda^{2}-5 \lambda+1}{2 \lambda^{2}(1-\lambda)}-\frac{6 \lambda^{2}-5 \lambda+1}{2 \lambda^{2}(1-\lambda)}(4 t+1), & t \in\left(-\frac{1}{4}, 0\right],\end{cases}
$$

where $\lambda$ is a root of the equation $F(\lambda)=4 \lambda^{3}-6 \lambda^{2}-3 \lambda+1=0 \lambda \in(0,1)$. There exists such a root because $F(0)=1>0$ and $F(1)=-4<0$.

Define the function $y(t)$ in $\left[-\frac{1}{2}, \infty\right)$ as

$$
y\left(t+\frac{1}{2}\right)=y(t)-z(t), \quad t \in\left[-\frac{1}{2}+\frac{k}{2}, \frac{k}{2}\right], \quad k=0,1,2, \ldots,
$$


where

$$
\begin{gathered}
y(t)=\varphi(t), \quad t \in\left[-\frac{1}{2}, 0\right], \\
z(t)= \begin{cases}\lambda^{n-2}\left(\frac{1}{4}-\frac{\lambda}{2}\right)-\lambda^{n-1}\left(t-n+\frac{1}{2}\right), & t \in\left[n-\frac{1}{2}, n\right), \\
\lambda^{n-2}\left(\frac{1-4 \lambda+2 \lambda^{2}}{4}\right)-\lambda^{n}\left(t-n+\frac{1}{2}\right), & t \in\left[n, n+\frac{1}{2}\right) .\end{cases}
\end{gathered}
$$

As in Example 1 it is proved that $y(t)$ is a solution of equation (15) with initial function $\varphi(t)$. We shall prove that $y(t)>0, t \in\left[-\frac{1}{2}, \infty\right)$, and $\limsup _{t \rightarrow \infty} y(t)>$ 0 .

Analogously to the considerations in Example 1 it is shown that $y(n)=\lambda^{n}$, $n=0,1,2 \ldots$ From equation $(7)$ it follows that

$$
y\left(n+\frac{1}{2}\right)=\lambda^{n}\left(\frac{1}{4}+\frac{\lambda}{2}\right), \quad n=0,1,2 \ldots
$$

Since the function $y(t)$ is linear in each interval $\left[-\frac{1}{2}+\frac{k}{4},-\frac{1}{4}+\frac{k}{4}\right]$, then in order to prove that $y(t)>0$, it suffices to prove that $y\left(-\frac{1}{2}+\frac{k}{4}\right)>0, k=0,1,2 \ldots$ Obviously, $y(n)>0$ and $y\left(n+\frac{1}{2}\right)>0$. It remains to show that $y\left(-\frac{1}{4}+\frac{n}{2}\right)>0$. The following inequalities are valid:

$$
\begin{gathered}
z\left(-\frac{1}{4}+n\right)=y\left(-\frac{1}{4}+n\right)-y\left(\frac{1}{4}+n\right), \\
z\left(\frac{1}{4}+n\right)=y\left(\frac{1}{4}+n\right)-y\left(\frac{3}{4}+n\right), \\
z\left(\frac{3}{4}+n\right)=y\left(\frac{3}{4}+n\right)-y\left(\frac{5}{4}+n\right) .
\end{gathered}
$$

From the above equalities it follows that

$$
\begin{gathered}
y\left(-\frac{1}{4}+n\right)-y\left(-\frac{1}{4}+n+1\right)=z\left(-\frac{1}{4}+n\right)+z\left(\frac{1}{4}+n\right), \\
y\left(\frac{1}{4}+n\right)-y\left(\frac{1}{4}+n+1\right)=z\left(\frac{1}{4}+n\right)+z\left(\frac{3}{4}+n\right) .
\end{gathered}
$$

We define the sequences $\left\{a_{n}\right\}$ and $\left\{b_{n}\right\}$ as

$$
a_{n}=y\left(-\frac{1}{4}+n\right), \quad b_{n}=y\left(\frac{1}{4}+n\right), \quad n=0,1,2 \ldots
$$

The inequalities (16) and (17) take the form

$$
a_{n}-a_{n+1}=z\left(-\frac{1}{4}+n\right)+z\left(\frac{1}{4}+n\right),
$$




$$
b_{n}-b_{n+1}=z\left(\frac{1}{4}+n\right)+z\left(\frac{3}{4}+n\right) .
$$

From the definition of $z(t)$ we obtain

$$
\begin{aligned}
& z\left(-\frac{1}{4}+n\right)+z\left(\frac{1}{4}+n\right)=\frac{\lambda^{n-2}}{4}\left(-\lambda^{2}-7 \lambda+2\right), \\
& z\left(\frac{1}{4}+n\right)+z\left(\frac{3}{4}+n\right)=\frac{\lambda^{n-2}}{4}\left(-4 \lambda^{2}-3 \lambda+1\right) .
\end{aligned}
$$

Hence the sequences $\left\{a_{n}\right\}$ and $\left\{b_{n}\right\}$ satisfy the nonhomogeneous difference equations

$$
\begin{aligned}
& a_{n}-a_{n-1}=\frac{\lambda^{n-1}}{4}\left(-\lambda^{2}-7 \lambda+2\right), \\
& b_{n}-b_{n-1}=\frac{\lambda^{n-1}}{4}\left(-4 \lambda^{2}-3 \lambda+1\right) .
\end{aligned}
$$

Sum up equalities (20) and (21) from 0 to $m-1$ and obtain

$$
\begin{aligned}
& a_{m}=a_{0}+\frac{\lambda^{2}+7 \lambda-2}{4} \sum_{k=0}^{m-1} \lambda^{k-2}, \\
& b_{m}=b_{0}+\frac{4 \lambda^{2}+3 \lambda-1}{4} \sum_{k=0}^{m-1} \lambda^{k-2} .
\end{aligned}
$$

For each $n \in N$ the following inequalities are valid:

$$
\begin{aligned}
& a_{n}>a_{0}+\frac{\lambda^{2}+7 \lambda-2}{4 \lambda^{2}(1-\lambda)}, \\
& b_{n}>b_{0}+\frac{4 \lambda^{2}+3 \lambda-1}{4 \lambda^{2}(1-\lambda)} .
\end{aligned}
$$

Since $a_{0}=\varphi\left(-\frac{1}{4}\right)=\frac{-2 \lambda^{3}+8 \lambda^{2}-5 \lambda+1}{4 \lambda^{2}(1-\lambda)}$, then

$$
a_{n}>\frac{-2 \lambda^{2}+17 \lambda-3}{4 \lambda(1-\lambda)}>0, \quad n \in N .
$$

From the equality $z\left(-\frac{1}{4}\right)=y\left(-\frac{1}{4}\right)-y\left(\frac{1}{4}\right)$ it follows that

$$
b_{0}=a_{0}-z\left(-\frac{1}{4}\right)=\frac{-4 \lambda^{3}+15 \lambda^{2}-8 \lambda+1}{4 \lambda^{2}(1-\lambda)} .
$$


Cosequently,

$$
b_{n}>\frac{\left(-4 \lambda^{2}+19 \lambda-5\right)}{4 \lambda(1-\lambda)}>0, \quad n \in N .
$$

Thus we have proved that the solution $y(t)$ constructed is strictly positive in the interval $\left[-\frac{1}{2}, \infty\right)$ and $\limsup _{t \rightarrow \infty} y(t)=0$.

Theorem 4. Let conditions $(A)$ hold, $q<0$ and $p>0(p \neq 1)$. Then, if $y(t)$ is a nonoscillating solution of equation $(1), \lim _{t \rightarrow \infty} y(t)=0$.

Proof. Let $y(t)>0$ finally. Then $z(t)$ is a finally positive strictly decreasing function. We shall consider the cases $(i) p \in(0,1)$ and $(i i) p>1$.

(i) Suppose that $\lim _{t \rightarrow \infty} z(t)=c>0$. We fix $\epsilon>0$ so that $\epsilon<c \frac{(1-p)}{(1+p)}$. The following inequality is finally valid.

$$
c<z(t)<c+\epsilon
$$

From Lemma 1 it follows that $\liminf _{t \rightarrow \infty} y(t)=0$. Choose a sequance $\left\{t_{n}\right\}$ so that $\lim _{n \rightarrow \infty} t_{n}=\infty$ and $\lim _{n \rightarrow \infty} y\left(t_{n}\right)=0$,

$$
y\left(t_{n}+\tau\right)=\frac{1}{p}\left(z\left(t_{n}\right)-y\left(t_{n}\right)\right)>\frac{1}{p}(c-\epsilon) .
$$

Consequently,

$$
z\left(t_{n}+\tau\right)>y\left(t_{n}+\tau\right)>\frac{1}{p}(c-\epsilon)>c+\epsilon
$$

(the last inequality follows from the choice of $\epsilon$. )But the inequality $z\left(t_{n}+\tau\right)>$ $c+\epsilon$ contradicts equation (22). Thus, $\lim _{t \rightarrow \infty} z(t)=0$ and since $y(t)<z(t)$, then $\lim _{t \rightarrow \infty} y(t)=0$.

(ii) Suppose that $\lim _{t \rightarrow \infty} z(t)=c>0$ and let $\limsup _{t \rightarrow \infty} y(t)=d$. Obviously, $d \leq c$. From Lemma 1 it follows that $\liminf _{t \rightarrow \infty} y(t)=0$, consequently $d=c$. Choose a sequance $\left\{t_{n}\right\}$ such that $\lim _{n \rightarrow \infty} t_{n}=\infty$ and $\lim _{n \rightarrow \infty} y\left(t_{n}+\tau\right)=c$. Passing to the limit as $n \rightarrow \infty$ in the inequality $z\left(t_{n}\right)>p y\left(t_{n}+\tau\right)$ we obtain $c \geq$ $p c>c$. The contradiction obtained shows that $\lim _{t \rightarrow \infty} z(t)=0$ and, consequently, $\lim _{t \rightarrow \infty} y(t)=0$. 
Remark 3. In the case $p=1$ and $q<0$, it is easily proved that each nonoscillating solution of equation (1) is bounded. From Lemma 1 it follows that $\liminf _{t \rightarrow \infty} y(t)=0$. We cannot claim, however, that $\lim _{t \rightarrow \infty} y(t)=0$. We shall illustrate this fact by the following example.

Example 3. Consider the equation

$$
\left(y(t)+y\left(t+\frac{1}{2}\right)\right)^{\prime}=-y(\gamma(t))
$$

with $\gamma_{0}=\frac{1}{2}$ and initial function $\varphi(t): \varphi(t)=1-2 t, t \in\left[-\frac{1}{2}, 0\right]$. Let $y(t)$ be the unique solution of equation (23) corresponding to $\varphi(t)$. We shall prove that $y(t)>0, t \in\left[-\frac{1}{2}, \infty\right)$ (i.e., $y(t)$ is a nonoscillating solution), $\liminf _{t \rightarrow \infty} y(t)=0$, and $\limsup _{t \rightarrow \infty} y(t)>0$.

From equation (8) it follows that the sequence $\left\{c_{n}\right\}_{n=1}^{\infty}\left(c_{n}=y(n)\right)$, satisfies the difference equatoin $c_{n+1}-\frac{1}{2} c_{n}=0$. Since $c_{0}=\varphi(0)=1$, then $c_{n}=\frac{1}{2^{n}}$, $n=0,1,2, \ldots$. Hence $\liminf _{t \rightarrow \infty} y(t)=0$. On the other hand, by induction we shall prove that $y\left(n+\frac{1}{2}\right)=1+\frac{1}{2^{n}}$ Integrating the equation $z^{\prime}(t)=-y(\gamma(t))$ from $\frac{-1}{2}$ to $t$, we obtain : $z(t)-z(0)=-\left(t+\frac{1}{2}\right)$ and from this it is follows that $y\left(t+\frac{1}{2}\right)=\frac{3}{2}+t$, hence $y(t)=1+t$ for $t \in\left[0, \frac{1}{2}\right)$ and for $t \rightarrow \frac{1}{2}$ we obtain that $y\left(\frac{1}{2}\right)=1+\frac{1}{2}$ so the assertion is valid for $n=0$.

Let we assume that $y\left(n-\frac{1}{2}\right)=1+\frac{1}{2^{n-1}}$ Then integrating (23) from $n-\frac{1}{2}$ to $n$ we obtain $z(t)-z\left(n-\frac{1}{2}\right)=\frac{-1}{2^{n-1}}\left(t-n+\frac{1}{2}\right)$ and from this it is immediatelly follows that $y\left(t+\frac{1}{2}\right)=1+\frac{3}{2^{n}}-\frac{t}{2^{n-1}}+\frac{n}{2^{n-1}}-\frac{1}{2^{n}}-y(t)$ and for $t \rightarrow n$ it is follows that $y\left(n+\frac{1}{2}\right)=1+\frac{1}{2^{n}}$ and the inductive step is complete.

Hence $\limsup y(t)>0$. Since $y(t)$ is a linear function in each interval $\left[n-\frac{1}{2}, n\right], \quad n=0,1,2, \ldots, y(n)>0$ and $y\left(n+\frac{1}{2}\right)>0$, then $y(t)>0$ for $t \in\left[-\frac{1}{2}, \infty\right)$.

\section{References}

[1] A. R. Aftebizadeh and J. Wiener, Oscillatory and periodic solutions of an equation alternately of retarded and advanced type, Apll. Anal. , 23 (1986), 219-231.

[2] A. R. Aftabizadeh, J. Wiener and J. Xu, Oscillatory and periodic solutions of delay differencial equations with piecewise constant argument, Proc. Amer. Math. Soc., 99 (1987), 673-679.

[3] M. U. Akhmet, Integral manifolds of differential equations with piecewise constant argument of generalized type, Nonlinear Analysis: Theory, Methods and Applications, 66, No. 2 (2007), 367-383. 
[4] M. U. Akhmet, Nonlinear Hybrid Continuous/Discrete-Time Models Atlantis Press, Amsterdam, The Netherlands, 2011.

[5] A. I. Alonso, J. Hong and R. Obaya, Almost periodic type solutions of differential equations with piecewise constant argument via almost periodic type sequences, Applied Mathematics Letters, 13, No. 2 (2000), 131-137.

[6] KUo-Shou Chiu, Periodic solutions for nonlinear integro-differential systems with piecewise constant argument, The Scientic World Journal (2014), 1-4.

[7] K. S. Chiu and M. Pinto, Oscillatory and periodic solutions in alternately advanced and delayed differential equations, Carpathian Journal of Mathematics, 29, No. 2 (2013), 149-158.

[8] K. S. Chiu and M. Pinto, Periodic solutions of differential equations with a general piecewise constant argument and applications, Electronic Journal of Qualitative Theory of Differential Equations, 46 (2010), 1-19.

[9] K. L. Cooke and J. Wiener, Retarded differential equations with piecewise constant delay, J. Math. Amal. Appl., 99 (1984), 265-297.

[10] K. I. Cooke and J. Wiener, An equation alternately of retarded an advanced type, Proceedings of the American Mathematical Society, 99 (1987), 726-732.

[11] I. Gyori and G. Ladas, Linearized oscillations for equations with piecewise constant arguments, Differential Integral Equations, 2 (1988), 123-131.

[12] I. Gyori, G. Ladas and L. Pakula, Conditions for oscillation of difference equations with applications to equations with piecewise constant arguments, SIAM J. Math. Anal., 22, No. 3 (1991), 269-773.

[13] I. Gyori and G. Ladas, Oscillations Theory of Delay Differential Equations, Clarendon Press, Oxford, 1991.

[14] J. K. Hale, S. M. V. Lunel, Introduction to Functional Differential Equations, SpringerVerlag, NewYork, 1993.

[15] Y. Huang, Oscillations and asymptotic stability of solutions of first order neutral differential equations with piecewise constant argument, J. Math. Anal. Apll., 149 (1990), $70-85$.

[16] V. Kolmanovskii, A. Myshkis, Introduction to the Theory and Application of Functional Differential Equations, Kluivert Academic Publishers, Dordrecht, 1999.

[17] A. D. Myshkis, On certain problems in the theory of differential equations with deviating arguments, Uspekhi Matematicheskikh Nauk, 32 (1977), 173-202.

[18] E. C. Partheniadis, Stability and oscillation of neutral delay differential equations with piecewise constant argument, Differential Integral Equations, 1 (1988), 459-472.

[19] M. Pinto, Asymptotic equivalence of nonlinear and quasi linear differential equations with piecewise constant arguments, Mathematical and Computer Modelling, 49, No-s: 9-10, (2009) 1750-1758.

[20] M. Pinto, Cauchy and Green matrices type and stability in alternately advanced and delayed differential systems, Journal of Difference Equations and Applications, 17, No. 2 , (2011) 235-254.

[21] S. M. Shah and J. Wiener, Advanced differential equations with piecewise constant argument deviations, Internat. J. Math. Math. Sci., 6 (1983), 671-703. 
[22] G. Wang Existence theorem of periodic solutions for a delay nonlinear differential equation with piecewise constant argument, Journal of Mathematical Analysis and Applications, 298, No. 1 (2004), 298-307.

[23] J. Wiener and A. R. Aftabizadeh, Differential equations alternately of retarded and advanced type, J. Math. Anal. Appl. , 129 (1988), 243-255.

[24] J. Wiener, Differential equations with piecewise constant delays, In: Trends in the Theory and Practice of Nonlinear Differential Equations (Ed. V. Lakshmikantham), Marcel Dekker, New York, (1983) 547-580.

[25] J. Wiener, Generalized Solutions of Functional Differential Equations, World Scientic, Singapore (1993). 